\title{
Stabilization of $\pi$-conjugated organoboron scaffolds by structural constraint
}

\author{
Tobias A. Schaub and Milan Kivala* \\ Department of Chemistry and Pharmacy, University of Erlangen-Nürnberg, \\ Henkestraße 42, 91054 Erlangen, Germany \\ E-mail: milan.kivala@fau.de
}

\begin{abstract}
Tricoordinate boron with its vacant p-orbital is a potent $\pi$-acceptor in conjugated organic molecules relevant to the construction of novel optoelectronic materials. The new concept of kinetic stabilization by structural constraint offers a powerful strategy that enables the synthesis of hitherto unprecedented boron-containing polycyclic aromatic hydrocarbons. These new planarized organoboranes feature intriguing photophysical and self-assembly properties and hence are particularly interesting for application in organic optoelectronic devices.
\end{abstract}

Keywords: Polycyclic aromatic hydrocarbons, organoboranes, kinetic stabilization, structural constraint

Owing to the increased environmental awareness and diminishing natural resources, the last decades have witnessed vigorous research activities in the field of energy-saving technologies with low environmental impact. In this regard, $\pi$-conjugated organic molecules emerged as one of the most promising candidates for the construction of innovative optoelectronic devices. ${ }^{1}$ The main reason is the ease of structural tuning by means of organic synthesis which allows for efficient tailoring of their photophysical properties, supramolecular behavior and thus of the resulting function.

A powerful approach to modify the nature of the parent $\pi$-conjugated systems is to incorporate heteroatoms into the polycyclic carbon-based scaffolds. ${ }^{2,3}$ Among the elements applicable to this methodology, boron is particularly qualified due to its electronic structure and its strategic position in the periodic table adjacent to carbon. Thereby, the intrinsic propensity of any tricoordinate boron species to reach the desired octet configuration is directly reflected in i) its pronounced Lewis acidity, ii) its trigonal planar geometry, and iii) the resulting effective orbital interaction of the vacant p-orbital on boron with the adjacent $\pi$-systems. Hence, a key issue for the construction of new trivalent organoboranes is to overcome their inherent sensitivity against moisture, oxygen and nucleophiles. 
In line with these characteristics, a variety of boron-containing functional materials with unique photophysical properties have been realized to date. ${ }^{4,5} \mathrm{~A}$ pertinent example is the donor- $\pi$-acceptor type (D- $\pi-A$ ) organoborane 1 disclosed by Wang et al. (Figure 1$){ }^{6}$ While this star-shaped molecule was shown to be an efficient blue emitter, its fluorescence was effectively quenched upon fluoride binding, suggesting its potential use in anion-sensing devices. The extended $\pi$-system 2 realized by Fang et al. demonstrated the significance of trivalent boron in A- $\pi-$ A type dyads for two-photon absorption. ${ }^{7}$ Recently, the triarylboryl motif has been combined with the inorganic $\left[\mathrm{Ru}(\mathrm{phen})_{3}\right]^{2+}$ complex (phen $=1,10$-phenanthroline) to provide the $\pi$-conjugated compound 3. ${ }^{8}$ This potential photosensitizer for solar energy conversion systems featured a low-energy emission in combination with a prolonged temperature-independent emission lifetime which was ascribed to the adjuvant effect of the triarylborane moiety on the observed metal-to-ligand charge-transfer (MLCT) interactions.
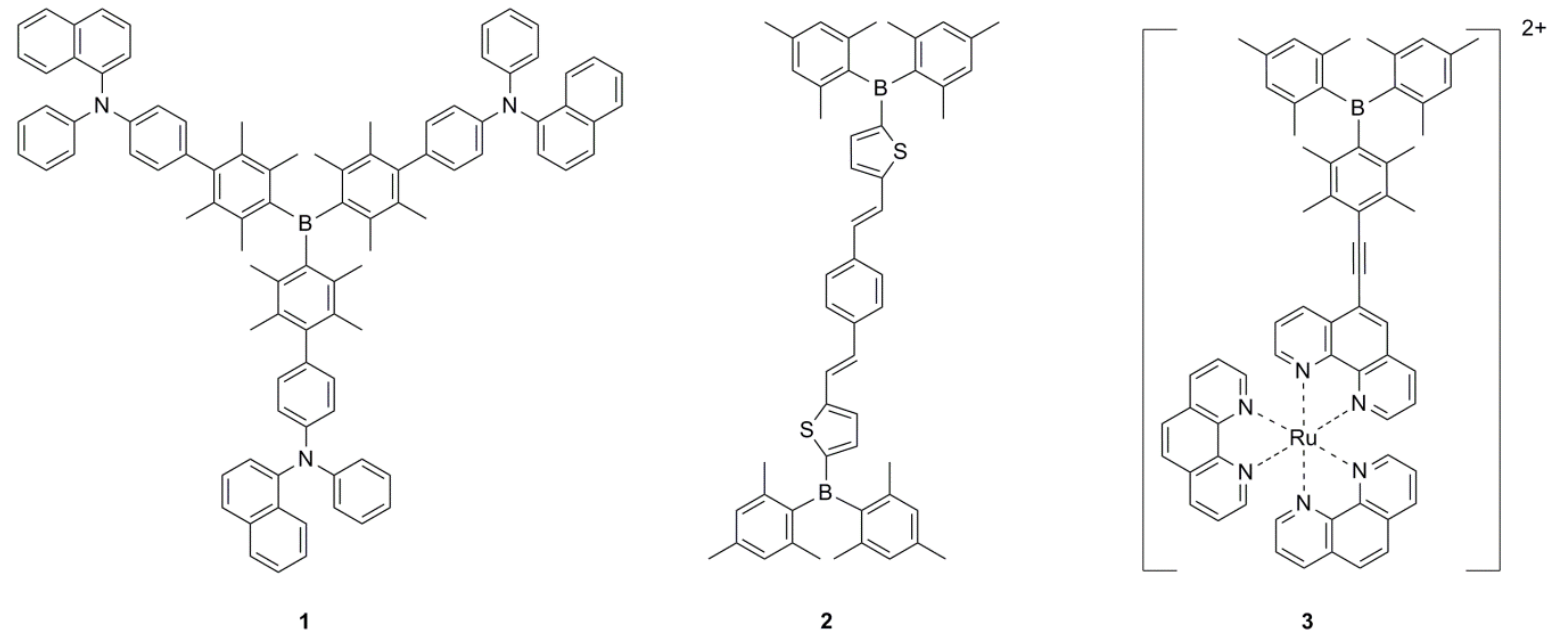

Figure 1. Functional $\pi$-conjugated systems 1-3 comprising the triarylboryl moiety as the electron-accepting unit.

To maintain the electron accepting nature of the boryl group, the formation of tetracoordinate organoborate Lewis acid-Lewis base complexes needs to be avoided. The common approach to stabilize the electron-deficient boron center relies on the attachment of sterically-protecting groups, such as duryl (2,3,5,6-tetramethylphenyl), ${ }^{6,8}$ mesityl (2,4,6trimethylphenyl, Mes), ${ }^{9}$ or 2,4,6-tri-tert-butylphenyl. ${ }^{10}$ In particular, the mesityl moiety is routinely employed to provide access to considerably air and moisture stable boron-containing compounds such as the triarylamine-based $\mathrm{D}-\pi-\mathrm{A}$ material $\mathbf{4}$ with interesting two-photon absorption characteristics reported by the groups of Müllen and Perry (Scheme 1). ${ }^{11}$ The synthesis of $\mathbf{4}$ involved an initial halogen-metal exchange reaction of an arylbromide, followed by the reaction with a suitable diarylboron electrophile, and is exemplary for the preparation of trivalent organoboron compounds. 

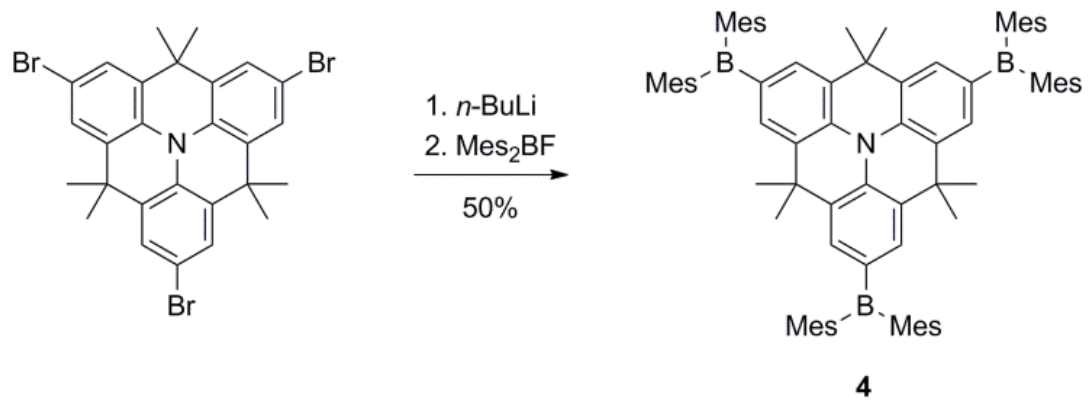

Scheme 1. Synthesis of the triarylamine-diarylboryl D- $\pi-A$ compound 4.

Adopting the isoelectronic relationship between the $\mathrm{B}-\mathrm{N}$ and $\mathrm{C}-\mathrm{C}$ units to $\pi$-conjugated macrocycles, ${ }^{12}$ Jäkle et al. recently reported the first ambipolar $\pi$-expanded borazine derivative $\mathbf{5}$ (Scheme 2). ${ }^{13}$ The synthesis of the macrocycle 5 was accomplished by cyclization of the borylated precursor $\mathbf{6}$ with stannylated 7 under pseudo-high dilution conditions, followed by treatment with triisopropylphenyl copper. Considerable electronic coupling between the nitrogen donor and the boron acceptor sites leads to a pronounced solvatochromic effect on the emission characteristics. The efficient electronic communication within the $\pi$-conjugated macrocycle $\mathbf{5}$ is further reflected in its higher oxidation and lower reduction potentials as compared to the electron-rich hexaaza- and electron-deficient hexaboracyclophane analogues, respectively.
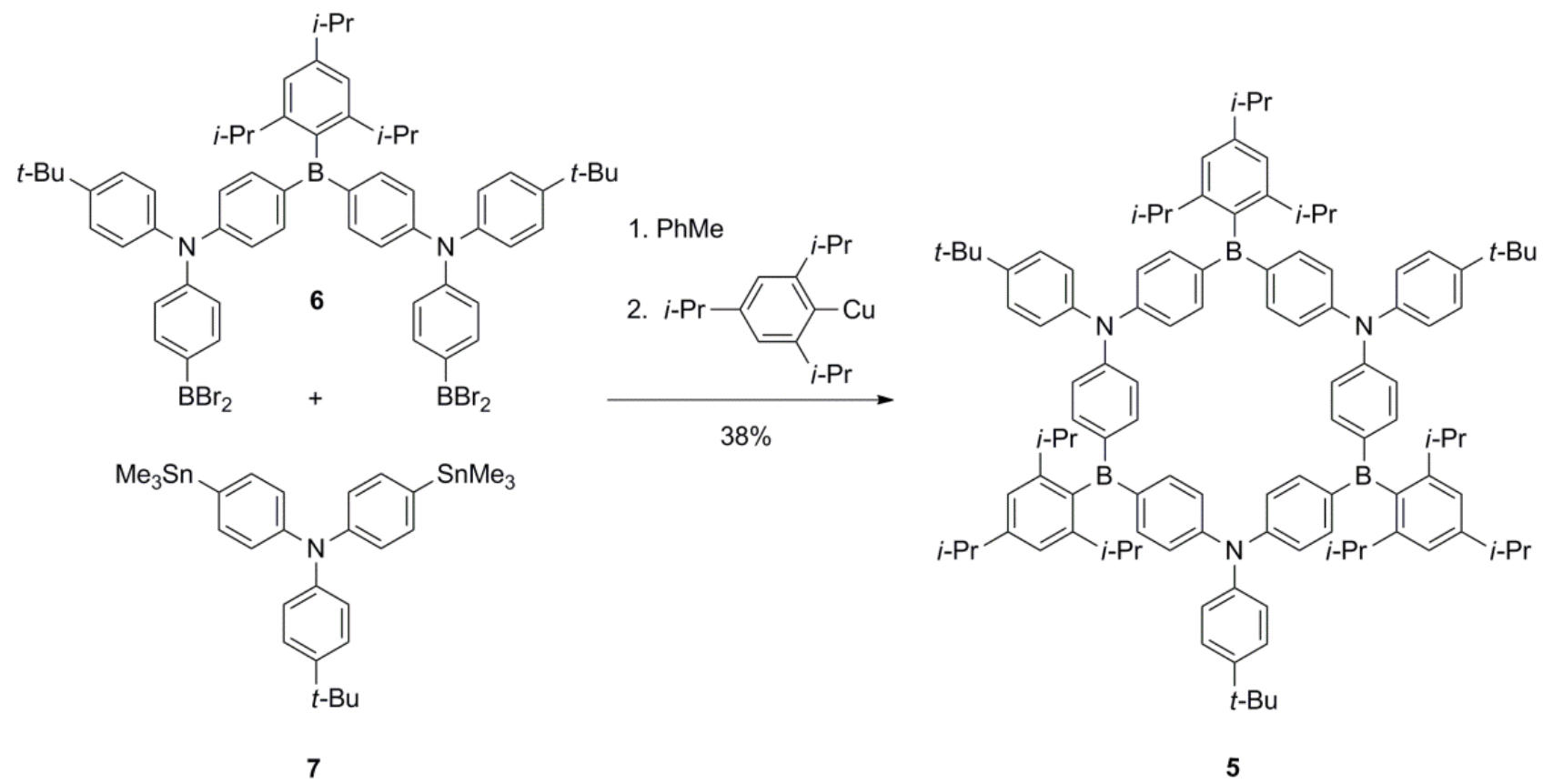

Scheme 2. Synthesis of the $\pi$-expanded borazine derivative 5 .

While the principal function of the bulky aryl substituents is the steric shielding of the sensitive boron center against undesired reactions, the enhancement of the electronic 
communication between the vacant p-orbital of boron and the neighboring $\pi$-systems is highly desirable as well. To this end, Yamaguchi et al. realized a new class of extended tri-9-anthrylboranes by a successive lithiation/quenching protocol starting from bromoanthracenes (Scheme 3). ${ }^{14}$ The highly electron-deficient dimesitylboryl-substituted compound $\mathbf{8}$ was stable towards air and moisture as a consequence of the steric demand of the anthryl and mesityl moieties. Despite the relatively large dihedral angles of about $53^{\circ}$ between the central borane plane and anthracene planes, spectroscopic and electrochemical data indicated a certain degree of $\pi$-conjugation extending through the central boron atom.

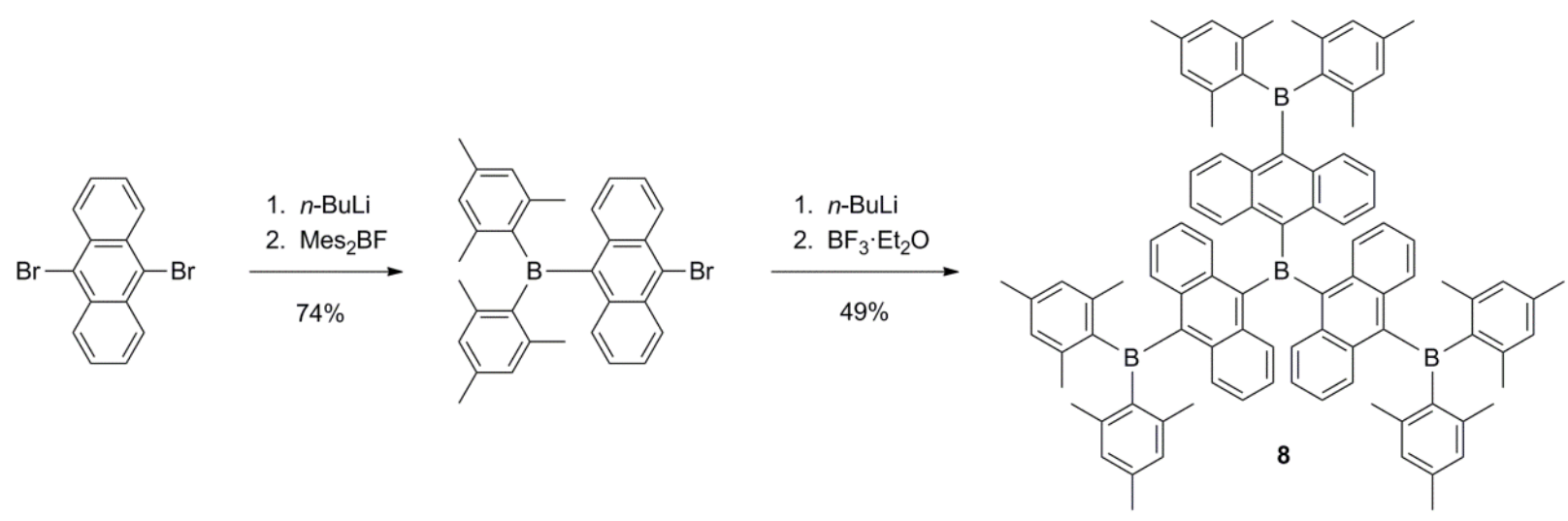

Scheme 3. Synthesis of the highly electron-deficient tri-9-anthrylborane $\mathbf{8}$.

The sterically demanding aryl substituents are beneficial to stabilize the Lewis acidic organoboranes, however, their propeller-like arrangement often results in partially disrupted $\pi$-conjugation across the boron atom and inefficient intermolecular electronic communication in the solid state. All this together has a detrimental effect on the device relevant chargetransporting performance of such materials. To overcome these severe drawbacks, Yamaguchi $e t$ al. envisaged the concept of kinetic stabilization by structural constraint by designing the planarized triphenylborane 9 (Scheme 4). ${ }^{15}$ The synthetic route towards 9 relied on the intramolecular Lewis acid-promoted cyclization of the key precursor 10 with $\mathrm{Sc}(\mathrm{OTf})_{3}$. Quite remarkably, the methylene-bridged intermediate 11 was oxidized with $\mathrm{CrO}_{3}$ in refluxing acetic acid to afford the planar carbonyl-bridged borane $\mathbf{1 2}$ in good yield, despite the harsh reaction conditions. The subsequent treatment of 12 with $\mathrm{ZnMe}_{2}$ and $\mathrm{TiCl}_{4}$ afforded the $D_{3 h}$-symmetric borane 9 in $76 \%$ yield. Although the tricoordinated boron center did not possess any steric protection in the vertical direction, compound $\mathbf{9}$ featured a remarkable chemical stability and could, for instance, be isolated by silica gel column chromatography in an open atmosphere. 


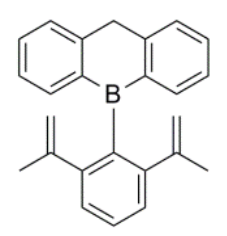

10

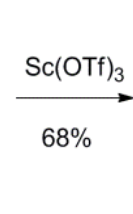

$68 \%$

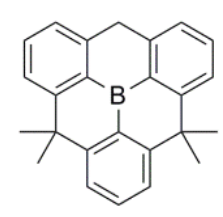

11
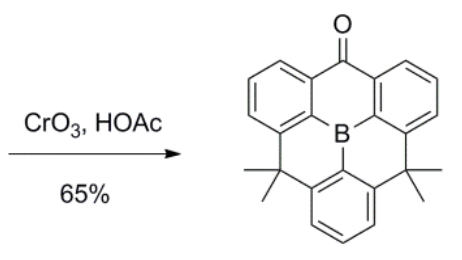

12
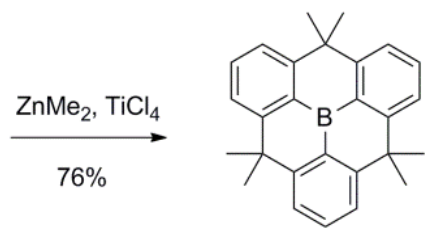

9

Scheme 4. Synthesis of the planarized triarylborane 9 .

$\mathrm{X}$-ray structural analysis confirmed that the $\mathrm{sp}^{3}$-hybridized dimethylmethylene tethers effectively enforced a nearly planar geometry of 9. The short B-C bond distances of $1.52 \AA$ pointed at the strong $\pi$-donation from the ipso-carbons to the central boron atom (Figure 2). This observation was further corroborated by the photophysical and electrochemical data indicating the extension of the $\pi$-system throughout the boron center. Consistently, the ${ }^{11} \mathrm{~B}$ NMR signal of $\mathbf{9}$ appeared upfield shifted at $48.6 \mathrm{ppm}$ compared to that of non-planarized triphenylborane (67.4 ppm). ${ }^{15}$ Although 9 was resistant to highly Lewis basic amines, it could still be converted to its stable fluoroborate complex with tris(dimethylamino)sulfonium difluorotrimethylsilicate (TASF). The tetracoordinated anionic complex could be transformed back to neutral 9 upon addition of stronger Lewis acidic $\mathrm{BF}_{3} \cdot \mathrm{Et}_{2} \mathrm{O}$. When going from tri- to tetracoordinated boron species, a reversible plane-to-bowl conversion was observed (Figure 2).
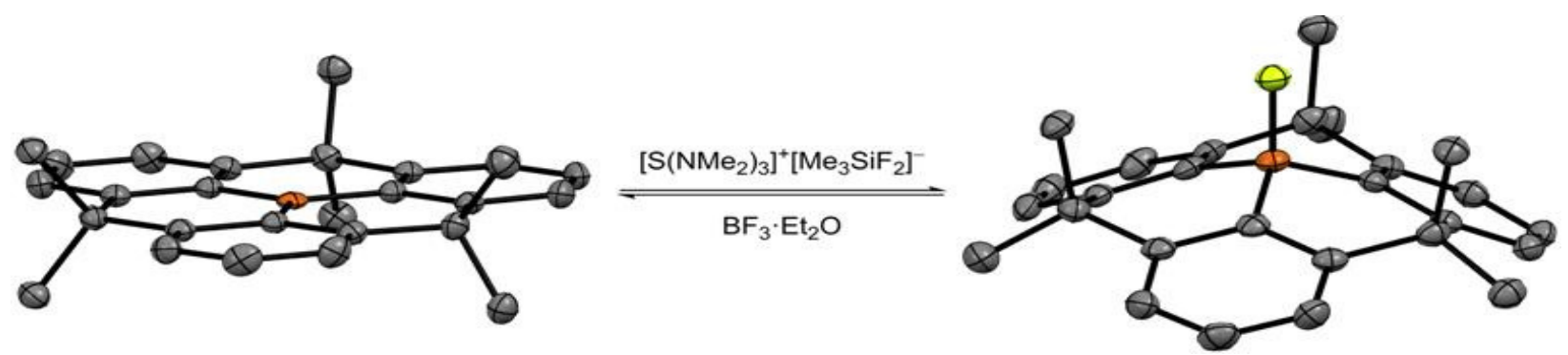

Figure 2. X-ray crystal structure of the planar triarylborane 9 (left) and its bowl-shaped fluoroborate complex (right). Color code: C-grey, B-orange, F-green; the countercation and hydrogen atoms are omitted for clarity.

The $\mathrm{sp}^{3}$-hybridized dimethylmethylene-bridges, however, disrupt full $\pi$-conjugation within the molecule and prevent columnar arrangement in the solid state. Thus, Yamaguchi et al. realized the fully planar boron-containing polyaromatic framework $\mathbf{1 3}$ by an intramolecular radical cyclization of $\mathbf{1 4}$, followed by oxidative cyclodehydrogenation of the partially cyclized precursor 15 with $\mathrm{FeCl}_{3}$ (Scheme 5). ${ }^{16}$ 
<smiles>COc1cccc2c(B(c3csc4cccc(Br)c34)c3csc4cccc(Br)c34)c3cccc(OC)c3cc12</smiles>

14
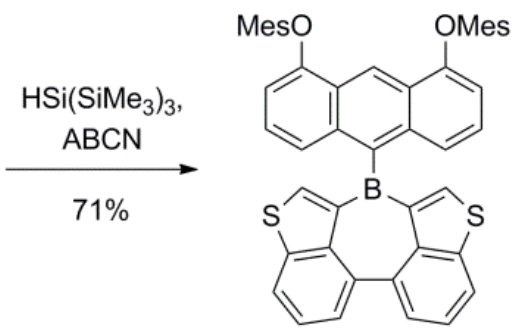

15

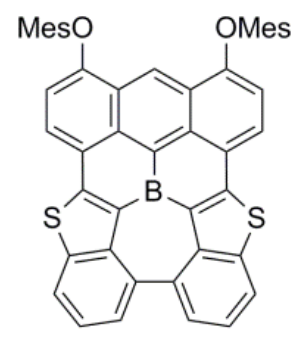

13

Scheme 5. Synthesis of the planar polyaromatic triarylborane 13. $A B C N=1,1^{\prime}$-azobis(cyclohexanecarbonitrile).

The bulky mesityloxy moieties were crucial to suppress the undesired intermolecular cyclization in the final step and to enhance the solubility of the target compound 13. In the single crystal packing, the virtually planar compound $\mathbf{1 3}$ occurs in face-to-face $\pi$-stacked dimers with a mean plane distance of $3.53 \AA$, which represents a hitherto unprecedented arrangement of a triarylborane-derived scaffold (Figure 3). As a consequence of the incorporation of the electronaccepting boron atom into the electron-rich polyaromatic skeleton, compound $\mathbf{1 3}$ showed broad absorption bands over the entire visible region originating from HOMO $\rightarrow$ LUMO and HOMO-1 $\rightarrow$ LUMO transitions. In analogy to dimethylmethylene-bridged 9, compound 13 still retains its intrinsic Lewis acidity and interacts with fluoride. However, the binding constant of $\mathbf{1 3}$ $\left(1.3 \times 10^{5} \mathrm{M}^{-1}\right)$ was slightly lower than that of $9\left(7.0 \times 10^{5} \mathrm{M}^{-1}\right)$ which nicely reflects the more rigid structure of 13. Apart from that, the formation of a labile pyridyl borate complex with interesting thermochromic behavior was observed in the presence of pyridine.

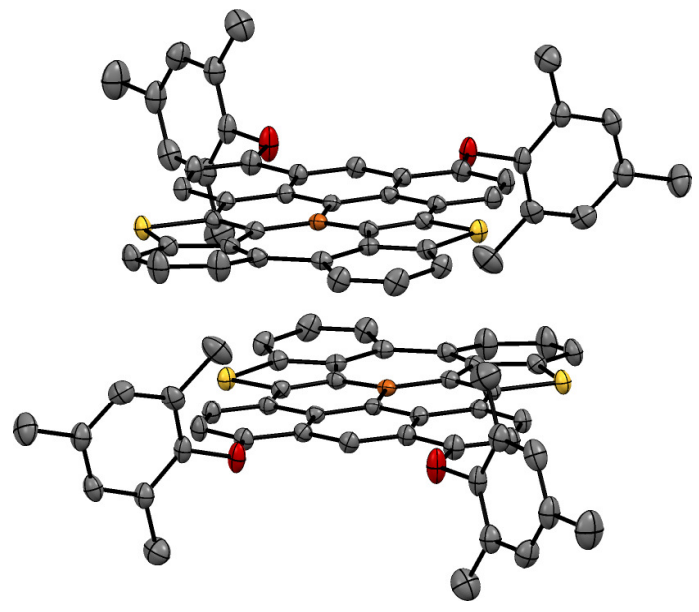

Figure 3. $\pi$-Stacked dimer in the single crystal packing of 13. Color code: C-grey, B-orange, O-red, S-yellow; hydrogen atoms are omitted for clarity. 
Only recently, the concept of kinetic stabilization by structural constraint was applied to the bottom-up synthesis of a boron-containing extended polycyclic aromatic hydrocarbon (PAH) 16, representing a molecular fragment of boron-doped graphene (Scheme 6). ${ }^{17}$ By replacing two of the internal ring junction carbon atoms in the parent all-carbon PAH by boron, Yamaguchi et al. avoided the generation of an unstable open-shell species. Thereby, each $\mathrm{C} / \mathrm{B}$ replacement corresponds to a formal one electron oxidation and hence the introduction of the two $\mathrm{B}$ atoms in 16 converts the formal all-carbon aromatic systems (50 $\pi$-electrons) into a formal antiaromatic one (48 $\pi$-electrons).

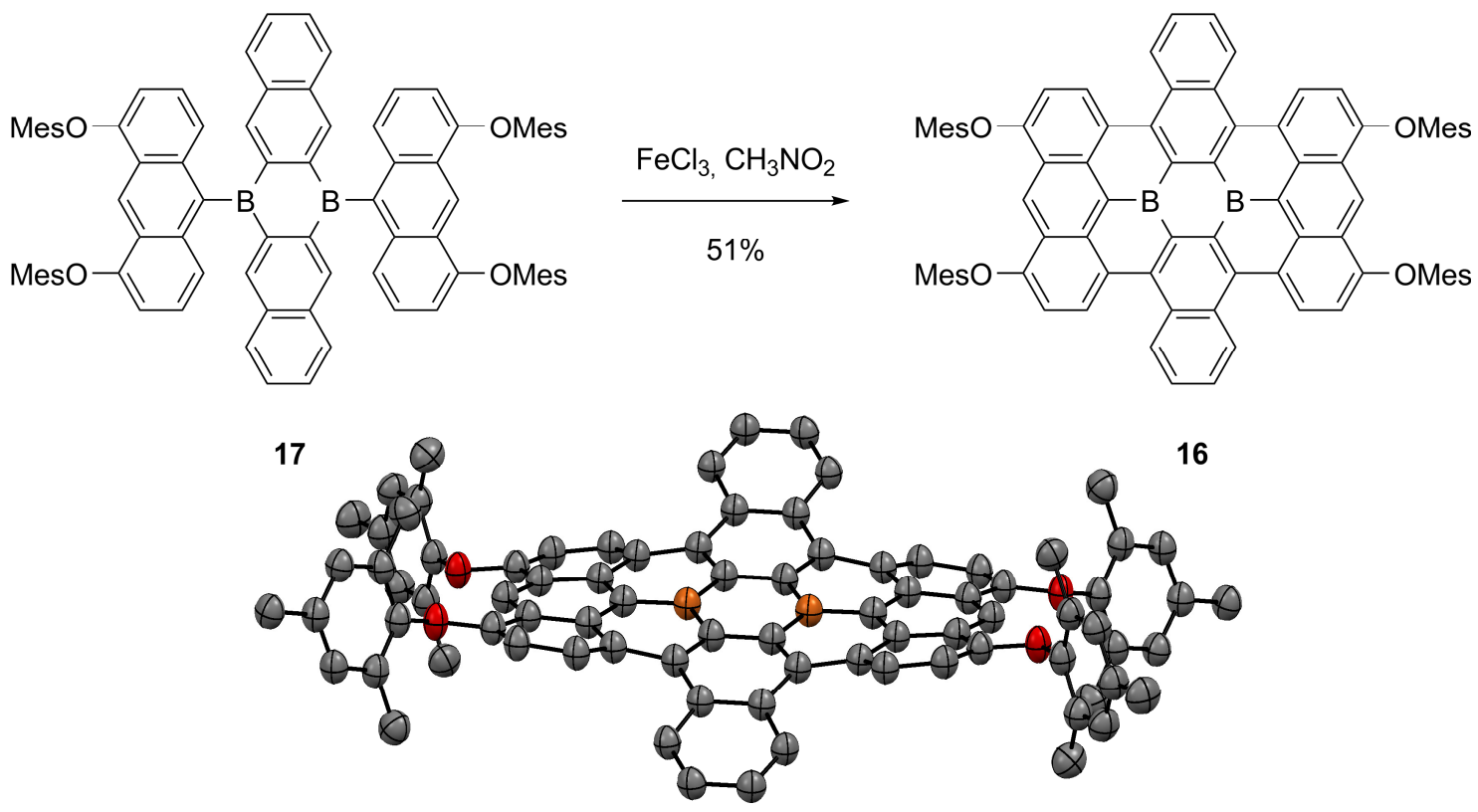

Scheme 6. Synthesis of the boron-doped contorted PAH 16. X-ray crystal structure of 16. Color code: C-grey, B-orange, O-red; hydrogen atoms are omitted for clarity.

The key step in the synthesis of the boron-doped PAH 16 was the $\mathrm{FeCl}_{3}$-mediated oxidative cyclodehydrogenation of the precursor 17. Again, the compound $\mathbf{1 6}$ was stable enough to be isolated by column chromatography on silica gel under air. Resulting from the steric congestion of the H-atoms in the four cove regions, the polyaromatic scaffold adapted a contorted geometry as revealed by X-ray crystallographic analysis. Similarly to dimethylmethylene-bridged 9, borondoped 16 was characterized by shortened B-C bonds lengths of 1.51-1.54 A. UV/vis absorption spectra of 16 showed broad absorption bands spanning over the entire visible region and a broad fluorescence band in the visible/near-IR region with a maximum wavelength at $679 \mathrm{~nm}$. From the comparison of the observed photophysical properties with those of the allcarbon congeners of similar size and periphery it was concluded that the introduction of boron atoms into the all-carbon polyaromatic scaffolds is a feasible way to shift the corresponding absorption and emission maxima towards lower energies, while maintaining their stability. Another prominent feature of the electron-deficient system 16 was the high stability to 
electrochemical reduction $\left(E_{\mathrm{red}, 1}=-1.45 \mathrm{~V}, E_{\mathrm{red}, 2}=-1.66 \mathrm{~V} v s . \mathrm{Fc}^{+} / \mathrm{Fc}\right)$ which disclosed its potential application as an electrode material for Li-ion batteries.

Building on the innovative approach of kinetic stabilization by structural constraint to compensate for the inherent Lewis acidity and high reactivity of the boron center, hitherto unknown organoboranes with intriguing properties became available. The few examples of planarized organoboranes prepared so far impressively illustrate the great potential of this approach and hence should stimulate further research activities in this direction.

\section{Acknowledgements}

Financial support by the German Fonds der Chemischen Industrie (FCI), the Erlangen Graduate School of Molecular Science (GSMS), and the Deutsche Forschungsgemeinschaft (DFG) as part of SFB953 "Synthetic Carbon Allotropes" is gratefully acknowledged by the authors.

\section{References}

1. Special issue on " $\pi$-Functional Materials"; Brédas, J.-L., Marder, S. R., Reichmanis, E., Eds., Chem. Mater. 2011, 23, 309.

2. Kervey, S.; Aurisicchio, C.; Bonifazi, D. In Functional Supramolecular Architectures for Organic Electronics and Nanotechnology; Samorì, P.; Cacialli, F. Eds.; Wiley-VCH: Weinheim, 2011, p 233.

3. Dral, P. O.; Kivala, M.; Clark, T. J. Org. Chem. 2013, 78, 1894. http://dx.doi.org/10.1021/jo3018395

4. Entwistle, C. D.; Marder, T. B. Angew. Chem. Int. Ed. 2002, 41, 2927. http://dx.doi.org/10.1002/1521-3773(20020816)41:16<2927::AID-ANIE2927>3.0.CO;2-L

5. Jäkle, F. Chem. Rev. 2010, 110, 3985. http://dx.doi.org/10.1021/cr100026f PMid:20536123.

6. Hudson, Z. M.; Wang, S. Acc. Chem. Res. 2009, 42, 1584. http://dx.doi.org/10.1021/ar900072u PMid:19558183.

7. Liu, Z.-Q.; Fang, Q.; Cao, D.-X.; Wang, D.; Xu, G.-B. Org. Lett. 2004, 6, 2933. http://dx.doi.org/10.1021/ol048984a PMid:15330651.

8. Sakuda, E.; Ando, Y.; Ito, A.; Kitamura, N. Inorg. Chem. 2011, 50, 1603. http://dx.doi.org/10.1021/ic1020669 PMid:21280604.

9. Entwistle, C. D.; Marder, T. B. Chem. Mater. 2004, 16, 4574. http://dx.doi.org/10.1021/cm0495717

10. Wakamiya, A.; Mishima, K.; Ekawa, K.; Yamaguchi, S. Chem. Commun. 2008, 579. http://dx.doi.org/10.1039/b716107g PMid:18209795.

11. Makarov, N. S.; Mukhopadhyay, S.; Yesudas, K.; Brédas, J.-L.; Perry, J. W.; Proń, A.; Kivala, M.; Müllen, K. J. Phys. Chem. A 2012, 116, 3781. 
http://dx.doi.org/10.1021/jp211878u PMid:22429020.

12. Bosdet, M. J. D.; Piers, W. E. Can. J. Chem. 2009, 87, 8. http://dx.doi.org/10.1139/v08-110

13. Chen, P.; Lalancette, R. A.; Jäkle, F. Angew. Chem. Int. Ed. 2012, 51, 7994. http://dx.doi.org/10.1002/anie.201203788 PMid:22767496.

14. Yamaguchi, S.; Akiyama, S.; Tamao, K. J. Am. Chem. Soc. 2000, 122, 6335. http://dx.doi.org/10.1021/ja994522u

15. Zhou, Z.; Wakamiya, A.; Kushida, T.; Yamaguchi, S. J. Am. Chem. Soc. 2012, 134, 4529. http://dx.doi.org/10.1021/ja211944q PMid:22369126.

16. Saito, S.; Matsuo, K.; Yamaguchi, S. J. Am. Chem. Soc. 2012, 134, 9130. http://dx.doi.org/10.1021/ja3036042 PMid:22595007.

17. Dou, C.; Saito, S.; Matsuo, K.; Hisaki, I.; Yamaguchi, S. Angew. Chem. Int. Ed. 2012, 51, 12206.

http://dx.doi.org/10.1002/anie.201206699 PMid:23081889.

\section{Authors' Biographies}

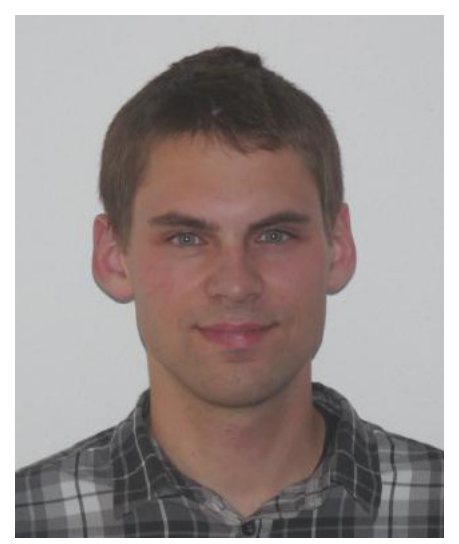

Tobias A. Schaub born 1986 in Erlangen, Germany, received both his B.Sc. degree (2010) and M.Sc. degree (2012) from the Friedrich-Alexander University of Erlangen-Nürnberg. Supported by the Fellowship of the German Fonds der Chemischen Industrie, he is currently pursuing his Ph.D. project under the supervision of Dr. Milan Kivala at the Friedrich-Alexander University of Erlangen-Nürnberg. His research activities focus on the synthesis of heteroatom-rich polycyclic aromatic hydrocarbons and the exploration of their physicochemical properties for application in optoelectronic devices. 


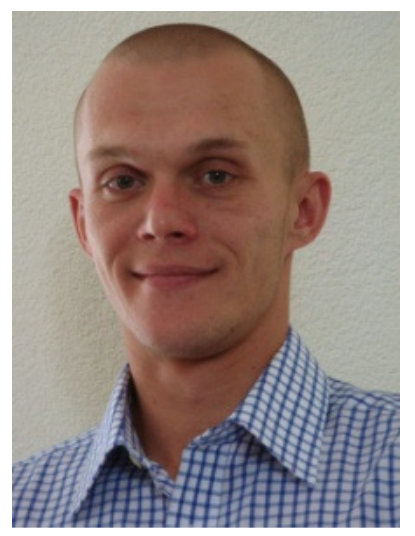

Milan Kivala born 1979 in Rakovník, Czech Republic, received his M.Sc. degree in 2003 from the Institute of Chemical Technology in Prague. In 2008, he completed his Ph.D. studies under the supervision of Prof. François Diederich at the ETH Zürich, focused on the synthesis of functional carbon-rich architectures based on acetylenic scaffolding. After a short postdoctoral stay at the same institution, he joined in 2009 the group of Prof. Klaus Müllen at the Max Planck Institute for Polymer Research in Mainz, Germany, where he was dealing with liquid crystalline polycyclic aromatic hydrocarbons. In 2011, he resumed his current position at the FriedrichAlexander University of Erlangen-Nürnberg as Habilitand associated with the chair of Prof. Rik Tykwinski. The focal point of his research is the chemical synthesis of heteroatom-doped polyaromatic $\pi$-systems for both fundamental studies and device applications. 